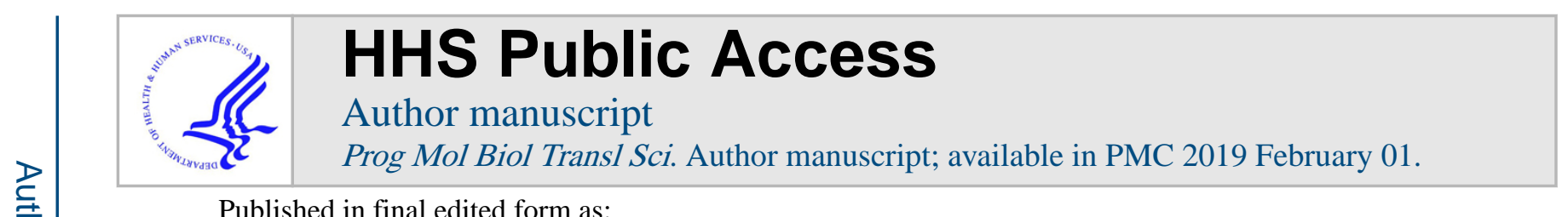

Published in final edited form as:

Prog Mol Biol Transl Sci. 2018 ; 155: 85-107. doi:10.1016/bs.pmbts.2017.12.003.

\title{
Cellular Metabolism and Aging
}

\author{
Andre Catic \\ Huffington Center on Aging; Stem Cells and Regenerative Medicine Center; Dan L. Duncan \\ Comprehensive Cancer Center - Program in Cell and Gene Therapy; Department of Molecular \\ and Cellular Biology; Baylor College of Medicine, Houston, TX, USA
}

\begin{abstract}
Metabolic changes are hallmarks of aging and genetic and pharmacologic alterations of relevant pathways can extend life span. In this review, we will outline how cellular biochemistry and energy homeostasis change during aging. We will highlight protein quality control, mitochondria, epigenetics, nutrient-sensing pathways, as well as the interplay between these systems with respect to their impact on cellular health.
\end{abstract}

\section{Keywords}

Mitochondria; Proteostasis; Nutrient-sensing pathways; Epigenetics

\section{Introduction}

"So Nature deals with us, and takes away our playthings one by one..." from "Nature" by Henry Wadsworth Longfellow Virtually every aspect of cell biology is affected by aging. Cellular aging is the result of physical and chemical assaults, of molecular exhaustion, and of physiological attempts to compensate for damage. Examples include toxic adducts to macromolecules, attrition of chromosomes, DNA damage, and impaired organelles ${ }^{1,2}$. Current pharmacological attempts to attenuate aging aim at stimulating cellular repair or at eliminating dysfunctional cells.

Evolutionarily conserved pathways with their built-in redundancies and overlaps appear more resilient to damage. In contrast, phylogenetically newer functions are more vulnerable and developmentally advanced cells seem at a disadvantage when it comes to aging. This vulnerability becomes evident in the functional decline of specialized cells of the adaptive immune system or of cortical neurons, which are more sensitive to age-induced damage than their primal and more resilient counterparts such as myeloid or mesenchymal cells.

Whereas cells of different types may age at different rates, consistent metabolic changes are observed across cell types ${ }^{3,4}$. In this review, we will discuss key metabolic pathways with relevance for cellular aging and outline how they are connected. Though the effects of aging and metabolism are intertwined, their relationship does not follow a scalable action-andreaction pattern. Instead, low levels of metabolic impairment can diminish the effects of aging through stimulation of repair systems, a concept known as hormesis, while severe metabolic injury accelerates aging. 


\section{Mitochondria and aging}

Mitochondria are the remnants of proteobacteria that became endosymbionts 1-2 billion years ago. Mitochondria still retain a small genome that encodes 37 genes in animals but is larger in simpler eukaryotes. The integration of mitochondrial genes into the host's chromosomes comes with advantages as their expression, replication, and repair are under control by the more sophisticated nuclear machinery of eukaryotes. The probable reason why mitochondria retained a small genome has to do with the topology of the encoded proteins: they are part of the respiratory chain complex and translation inside the mitochondrial matrix allows for proper insertion into the inner mitochondrial membrane, whereas nuclear encoded proteins are translated in the cytosol and may require import through the outer and inner mitochondrial membranes ${ }^{5}$.

In contrast to their neglect of DNA housekeeping, mitochondria have become the central hub for metabolism of the host cells. They not only produce ATP, but are also essential for the conversion of nutrients into building blocks (intermediary metabolism), for signaling, and for apoptosis. One of the most exciting areas of research involves the regulation of nuclear gene expression by mitochondria through "retrograde communication". While this pathway is well-defined in yeast, it lacks conserved components in higher eukaryotes and how the expression of nuclear encoded mitochondrial genes is adjusted to respiratory activity in mammalian cells is under intense investigation ${ }^{6,7}$. Apart from activation or silencing of specific genes, mitochondria also control nuclear activity through metabolites that influence the epigenetic landscape (see 5. Coordination of nuclear activities and metabolism).

Mitochondrial diseases have classically been defined as based on mutations of the mitochondrial genome. However, the majority of the more than 1,000 mitochondrial proteins are encoded in the nucleus and not inherited strictly maternally ${ }^{8}$. The list of diseases that are based on nuclear encoded mitochondrial disorders is growing, as is our understanding of the involvement of this organelle in basic and complex cellular functions. In addition to metabolic dysfunction caused directly by mitochondrial impairment, several diseases are also indirectly influenced by mitochondrial activity, for instance neurodegenerative diseases and cancer. Given their dominant role in metabolism, it is not surprising that mitochondria also play a central role in the aging process ${ }^{9-11}$.

\section{ROS theory of aging}

One of the most widely published theories of aging involves reactive oxygen species $(\mathrm{ROS})^{12}$. These molecules are byproducts of the respiratory chain in the inner mitochondrial membrane, where redox reactions create a proton gradient that drives ATP generation. Superoxide radicals are produced by complex I and III of the respiratory chain. Three scavenging mechanisms, superoxide dismutase (SOD; mitochondrion and cytosol), catalase (peroxisome) and glutathione peroxidase (mitochondrion and cytosol) act in a stepwise fashion to remove these radicals. First, SOD detoxifies the superoxide and creates hydrogen peroxide. This intermediary can form the more dangerous hydroxyl radical if not removed. Catalase and glutathione peroxidase prevent hydroxyl radical formation by converting hydrogen peroxide into water. Increased SOD activity without changes to catalase or glutathione peroxidase can be harmful due to accumulation of damaging hydroxyl radicals. 
In contrast, elevated levels of glutathione promote glutathione peroxidase's reducing activity and can have beneficial health effects. Patients who were fed bioavailable precursors of glutathione had significantly improved laboratory and clinical parameters in the HIV model of accelerated aging ${ }^{13-15}$. Whether increasing glutathione through supplementation with the precursor amino acids cysteine and glycine will increase life span in this clinical model remains to be seen.

Increased ROS production through desynchronized respiration and/or lower ROS scavenging capacity with age offers an attractive model to explain damage to proteins through carbonylation or to DNA through guanine modifications ${ }^{16,17}$. With their proximity to the respiratory chain, mitochondrial proteins and mitochondrial DNA that lacks protective nucleosomes are especially prone to damage. Indeed, mice with error-prone mitochondrial DNA replication age prematurely 18,19 . However, the theory of ROS-induced aging has been reevaluated over the past decade. Genetic alterations in ROS scavengers or treatment with antioxidants failed to show consistent effects on life span regulation ${ }^{20}$. Different species' life expectancies do not correlate with ROS levels. Moreover, there is evidence that modest levels of ROS have an anti-aging effect by triggering a stress-relieving response (hormesis). Combined, it appears that the dosage is critical when evaluating ROS in the context of aging, but reduction of ROS alone does not extend life span under physiological conditions.

\section{Mitochondrial aging}

Mitochondria replicate independently of the cell cycle. In addition, they undergo fusionfission cycles, which serve to adapt ATP production levels, allow for repair of mitochondrial DNA damage, and adjust the level of heteroplasmy (i.e. the degree to which multiple different mitochondrial DNA species coexist in the same cell) ${ }^{21}$. Mitochondrial removal by autophagy also requires fission of the organelle (see 3. Proteostasis network). Aged cells have impaired fusion and fission, thus reducing overall metabolic flexibility. As a consequence, mitochondria fail to adapt to nutrient availability or signaling cues and switch between oxidization of the substrates fatty acids, glucose, and amino acids ${ }^{22}$.

There is solid evidence that mitochondrial activity declines with age in tissues as well as in individual cells ${ }^{23}$. Well documented types of damage include replication errors of mitochondrial DNA and toxic adducts on mitochondrial proteins. The elevated $\mathrm{pH}$ and high levels of acetyl-CoA and succinyl-CoA in the mitochondrial matrix promote non-enzymatic acylation of $\varepsilon$-amino groups. These lysine modifications can prove harmful to the function of proteins. The removal of such adducts is catalyzed by sirtuins, a class of protein deac(et)ylases that are particularly enriched in mitochondria (SIRT3, SIRT4, and SIRT5). Their activity improves mitochondrial function and contributes to healthy aging, though not necessarily to extended life expectancy ${ }^{24}$. Sirtuins require $\mathrm{NAD}^{+}$as coenzyme and this metabolite is a key anti-aging effector (see 4. Nutrient-sensing pathways). In addition to the mitochondrial sirtuins, SIRT1 promotes mitochondrial activity in the cytosol by deacetylating the transcriptional coactivator PGC1a, allowing it to promote the expression of NRF-1 target genes. NRF-1 is a master transcription factor of numerous nuclear encoded mitochondrial genes. PGC1a, together with increasing levels of ROS, also stimulates NRF-2, which activates a support pathway and increases the expression of anti-oxidant 
stress response genes ${ }^{25}$. Combined, sirtuins increase mitochondrial activity and preserve their function by adapting the stress response (Figure 1). SIRT1 and SIRT3 in particular have been linked to maintaining metabolic health, though initial findings on their ability to extend life span have been disproven ${ }^{26,27}$.

\section{Proteostasis network}

In many cells, protein synthesis and removal are the most resource-consuming processes, tightly connecting them to energy metabolism. Furthermore, proteins are the main executors of cell function and dysregulated production or protein dysfunction have been implicated in aging. In particular, the highly specialized neurons of the mammalian brain seem to be sensitive to protein damage ${ }^{28}$. The expression of aggregation-prone polypeptides and the inability of these cells to divide and dilute protein accumulation are the root causes of several neurodegenerative diseases. Protein stress by itself may not damage neurons, but makes these frail cells more susceptible to a second toxic injury, such as inflammation or metabolic insults. Apart from neurons, most cells show consistent changes to protein homeostasis (proteostasis) with age, such as impaired proteasome and chaperone function $^{29-34}$.

\section{Translation control}

Proteostasis involves protein synthesis, structural and topological changes, and polypeptide removal. Lowering the biogenesis of polypeptides indeed alleviates stress and inhibition of global translation has positive effects on life span extension ${ }^{35}$. Experimental reduction of translation includes genetic manipulation of ribosomes or translation factors, as well as pharmacological interventions, most notably through inhibition of the mTOR signaling pathway (see 4. Nutrient-sensing pathways). Cell stress also reduces overall protein synthesis and the reduction of translation is accomplished by phosphorylation of the translation initiation factor eIF2 $a$ by kinases such as GCN2 and PKR. However, select proteins may be translated at higher rates under these conditions as their synthesis is either independent of eIF2a or even inhibited by native eIF $2 a^{36}$. Among them is the transcription factor ATF4 that drives the integrated stress response (ISR) that favors expression of life span-extending factors such as transcription factors and executors that deal with oxidative damage. This longevity-promoting effect of the ISR is also triggered by caloric restriction, the most successful intervention thus far to improve longevity and health span.

\section{UPR}

The main purpose of the unfolded protein response (UPR) is to reduce protein stress in the endoplasmic reticulum (ER) by altering protein production in a manner that is similar and partially overlapping with the ISR (Figure 2$)^{37}$. Triggered by misfolding of proteins within the ER, the UPR attenuates global translation through phosphorylation of initiation factor eIF2 $a$ by the ER-resident kinase PERK. A second arm of the UPR involves nuclear translocation of the transcription factor ATF6, which increases expression of ER-resident chaperones. Third, activation of the RNase IRE1 leads to alternative splicing of the transcription factor XBP1, which alters the proteostatic milieu in the $\mathrm{ER}^{38}$. Slight activation of the UPR, in particular higher expression of XBP1, has beneficial effects on survival and 
life $\operatorname{span}^{37}$. Interestingly, XBP1 also acts in a cell non-autonomous manner as a stress sensor in the nervous system of nematodes. Upregulation of XBP1 in neurons leads to UPR activation in peripheral organs ${ }^{39}$.

\section{Chaperone network}

Protein synthesis requires 30-50\% of cellular energy and is even under optimal conditions error-prone. By some estimates, a third of polypeptides fail to fold properly during translation in mammalian cells ${ }^{40-43}$. From the cradle to the grave, a network of chaperones supports protein folding and activity. Initially, ribosome-associated chaperones and foldases ensure proper structural assembly of a newly synthesized polypeptide. The nascent polypeptide-associated complex (NAC) accounts for most of the chaperone-assisted protein folding of newly translated proteins ${ }^{44}$. A second protective layer is provided by chaperones such as HSP70 and HSP40, which fold proteins in an ATP-dependent manner. Finally, if nascent proteins require additional assistance, they are transferred into chaperonins, closed nanocages that allow protein folding in isolation without interference from the otherwise crowded protein environment in the cytosol ${ }^{45}$. Failure to fold proteins eventually leads to their removal through the ubiquitin-proteasome system (UPS) or autophagy (see below).

Sentinel chaperones such as HSP90 scan the mature proteome for misfolded polypeptides. These chaperones can attempt to refold client proteins or direct them for removal. Approximately 332 chaperones and associated proteins are encoded in the human genome ${ }^{46}$. This "chaperome" consists of constitutive elements but is also enriched for stress response factors that are upregulated by heat shock or by the transcription factors ATF4 (for the ISR) and ATF6 (for the UPR). The dynamic response to protein stress is reduced with age and transgenic overexpression of chaperones improves life $\operatorname{span}^{47-49}$. However, it should be noted that abnormally high levels of chaperones come at a cost: they dampen signaling by the heat shock transcription factor HSF1 through feedback mechanisms and eliminate the ability to mount a proper stress response ${ }^{50}$. Further, overexpressed chaperones can soak up client polypeptides and thus reduce the bioavailability of critical proteins.

\section{UPS}

The UPS (ubiquitin-proteasome system) is the primary pathway to eliminate proteins in a specific fashion. A decrease of UPS activity with age has been linked to deficiencies in proteostasis. Further, one of the functions that distinguishes the long-lived naked mole rat from shorter lived rodents is the robustness of its UPS ${ }^{51,52}$. Similarly, healthy centenarians have been shown to maintain high proteasomal activity ${ }^{53}$.

Proteins that are destined for degradation by the proteasome are first tagged with several copies of the small polypeptide ubiquitin in a chain-like fashion. The covalent attachment of ubiquitin to target proteins occurs through a series of enzymatic events, involving ubiquitinactivating enzymes, conjugating enzymes, and finally ubiquitin ligases. Approximately 600 ubiquitin ligases exist in human cells. These ligases can associate with hundreds of different substrate recognition domains, allowing for a substantial combinatorial diversity that ensures specificity of the tagging process ${ }^{29}$. Similar to the chaperone network, several proteins of the UPS are upregulated upon stress. For instance, the UPR activates ubiquitination enzymes 
that allow for the specific extraction, ubiquitination, and proteasomal degradation of ERresident proteins in a process called ERAD (Endoplasmic reticulum-associated protein degradation). The UPS also regulates chaperone activity with ubiquitin ligases such as CHIP (STUB1), which tags chaperone client proteins for degradation during stress and ubiquitinates the chaperone HSP70 for removal during recovery from stress ${ }^{54}$. The ability of the UPS to target specific substrates also triggers the removal of organelles or bulk protein aggregates through autophagy (see below), where ubiquitination serves as a ligand for autophagosome receptors.

The UPS is a critical regulator of gene expression through the removal of short-lived transcription factors. Degradation therefore has wide-ranging effects on cellular activity and metabolism. One example of metabolic feedback mechanisms between mitochondria, the UPS, and gene activity involves ROS. These chemical radicals can dampen the activity of the UPS, which in turn stabilizes transcriptional repressors. This reduces the expression of nuclear encoded mitochondrial genes, attenuating oxidative metabolism. Replacement of carbonylated proteasome subunits through new rounds of transcription and translation, and the activation of transcription factor NRF-2, which leads to the production of ROS scavengers and executors of autophagy and the UPS, eventually normalize oxidative metabolism following ROS exposure $29,55,56$.

\section{Mitochondrial proteostasis}

Mitochondria have a select number of proteases and chaperones that are expressed in the cytosol and imported into the intermembrane or matrix chambers, or anchored to the membranes. These mitochondrial proteases ensure the import and activity of proteins into mitochondria, and maintain the homeostasis of the respiratory chain complex, which is partly encoded by the mitochondrial genome. Protein stress inside mitochondria triggers a mitochondrial version of the UPR (UPR ${ }^{\mathrm{mt}}$ ), which increases expression of chaperones, proteases, and ROS detoxifying factors ${ }^{57}$. The elements triggering the UPR ${ }^{\mathrm{mt}}$ are surprisingly different between species, but the response to stress appears overall conserved. The $\mathrm{UPR}^{\mathrm{mt}}$ is responsible for refolding or destroying misfolded proteins and thereby improving the efficiency and the health of mitochondria. Notably, a recent publication pointed out this stress response might also have deleterious effects on mitochondrial health. When healthy and damaged mitochondria coexist (heteroplasmy), the UPR ${ }^{\mathrm{mt}}$ can provide sufficient buffering capacity to maintain dysfunctional organelles, which, in the absence of $\mathrm{UPR}^{\mathrm{mt}}$, would have been competitively replaced by healthy mitochondria ${ }^{58}$. Regarding aging, the involvement of the UPR ${ }^{\mathrm{mt}}$ remains contested. Upregulation of some UPR ${ }^{\mathrm{mt}}$ elements promotes resilience to ROS and increases life span (mitohormesis), while other elements accomplish the opposite. More work will be necessary before a verdict can be reached on the role of the UPR ${ }^{\mathrm{mt}}$ in aging 59,60 .

Cross-talk between the mitochondrial and cytosolic proteostasis systems occurs at multiple layers and may also play an important role in aging (Figure 3). A dedicated cytosolic stress response exists to deal with mitochondrial proteins that have failed to enter the organelle and mitochondria-associated degradation involves the extraction of proteins from the organelle for cytosolic degradation by the UPS, in a mechanism resembling the UPR ${ }^{61,62}$. Further, 
mitochondrial dysfunction can elicit a cytosolic stress response that enhances the performance of non-mitochondrial proteins and increases cellular fitness ${ }^{63}$.

Mitochondria have also been suggested to assist cytosolic proteostasis by importing protein aggregates and digesting them with matrix proteases. This observation might explain how mitochondrial defects impact neurodegenerative diseases, not only through weakening the metabolism of neurons but also by failing to clear protein aggregates. Indeed, mitochondrial dysfunction accelerates the course of some protein aggregate diseases ${ }^{64}$. How these aggregated proteins mechanistically enter mitochondria and how this pathway affects aging and disease awaits further clarification ${ }^{65}$.

\section{Autophagy}

Autophagy is the proteostatic mechanism with the strongest ties to longevity. In simpler eukaryotes, life span extension by rapamycin requires induction of autophagy ${ }^{66,67}$. In addition, direct overexpression of regulators and executors of autophagy have been shown to have beneficial effects on health and life $\operatorname{span}^{68}$. Autophagy can be divided into three subtypes. First, chaperone-mediated autophagy targets individual proteins through interaction with heat shock proteins for lysosomal import and degradation. Second, microautophagy involves direct engulfment of proteins by the lysosome. The third, macroautophagy, is the most important mechanism for metabolic control and life span regulation ${ }^{69}$. Following initiation of the phagosome at mitochondrial-ER contact surfaces, a growing vesicle engulfs macromolecular complexes or organelles and eventually fuses to the lysosome for degradation. Macroautophagy is important for the recycling of lipids, sugars, amino acids, and metals, and its contribution to life span extension is based on the removal of damaged proteins, superfluous lipids, impaired organelles, and the regulation of inflammation and cell death.

An important aspect of macroautophagy is the removal of damaged or excess mitochondria. Mitochondrial dysfunction reduces the ability of these organelles to import proteins. The kinase PINK1 acts as an indicator of import stress and accumulates on the outer membrane. PINK1 phosphorylates ubiquitin and activates the ubiquitin ligase PARKIN. PARKIN conjugates a plethora of accessible mitochondrial proteins with phospho-ubiquitin, which attracts phagosome receptors for internalization of the organelle ${ }^{70,71}$. Mutations in this pathway lead to early onset Parkinson's disease. The control of mitochondrial numbers and the overall state of aerobic metabolism of cells is also regulated through mitophagy. One study exemplified how aged hematopoietic stem cells with increased autophagy display healthier, more juvenile features. The authors argued that removal of mitochondria and shifting of the overall metabolism towards glycolysis contributed to increased cellular health ${ }^{72}$. Apart from the engulfment of entire mitochondria, smaller portions can also be pinched off in vesicles containing oxidized proteins for lysosomal targeting in a process that is independent from autophagy or the fusion/fission cycle. These carriers are referred to as mitochondria-derived vesicles ${ }^{73}$.

The scale of autophagy has been studied best in the liver, where up to $70 \%$ of proteome turnover occurs through this mechanism. Inhibition of autophagy leads to the accumulation of poly-ubiquitinated inclusions, indicating that the proteasome system alone is insufficient for 
bulk protein removal, but instead functions mainly in selective polypeptide degradation or in ubiquitination as a recognition tag for the autophagosome. Autophagy has also been implicated in restructuring of the nuclear architecture. However, it remains to be seen whether nuclear autophagy is involved in the aging process ${ }^{74,75}$.

Lysosomes are the endpoint of phagosome breakdown and have remarkable capacity to extract and recycle individual nutrients and building blocks ${ }^{76}$. Recently, the role of lysosomes in the aging process has been extended to a lysosome-to-nucleus signaling function and additional lysosomal pathways with relevance for aging are likely awaiting discovery ${ }^{77}$.

\section{Nutrient-sensing pathways}

Pathways regulating energy metabolism and adaptation to nutrients are commonly associated with life span control. The main players are the insulin-IGF1 pathway, mTOR complexes, AMPK, and sirtuins (Figure 4). The mechanisms of these pathways have been covered in detail in a series of excellent reviews ${ }^{3,4,78-83}$. Here, we will briefly outline their function and their interplay with other cellular systems pertaining to life span regulation.

\section{Growth pathways}

The insulin-IGF1 pathway regulates growth in times of ample nutrient supply. Through intermediaries such as PI3K and Akt, this pathway suppresses FOXO transcription factors. FOXO transcription factors have multiple downstream effects: they activate autophagy, the UPS, and the ROS stress response, are essential for stem cell function, have tumorsuppressive activities, and promote life span extension ${ }^{84}$. Phosphorylation by Akt prohibits nuclear translocation of FOXOs and instead activates the mTOR complex ${ }^{85}$. The mTOR kinase complex is a master regulator of cell growth and mTOR activation increases protein synthesis, energy expenditure and induces a metabolic switch from frugality to anabolic processes that accelerate aging. The insulin-IGF1 pathway responds to high glucose levels, while mTOR responds directly to high amino acid concentrations and indirectly to glucose levels through Akt. To date, the mTOR inhibitor rapamycin has shown the most promising pharmacologic effect on life span extension in mammals. As mentioned above, the positive impact of rapamycin is autophagy-dependent. The other highly effective intervention, caloric restriction, slows cell growth by reducing both insulin-IGF1 and mTOR signaling ${ }^{80}$. However, the life span extension mediated by these interventions is limited and sustained inhibition of vital systems can become harmful, increase insulin resistance, lower cellular fitness, and accelerate aging over time ${ }^{3}$.

\section{Restrictive pathways}

Whereas insulin-IGF1 and mTOR represent anabolic "high energy" pathways, the catabolic "low energy" factors AMPK and sirtuins ${ }^{86,87}$ counteract mTOR and enhance mitochondrial activity to produce more ATP through fatty acid oxidation, and induce autophagy to recycle nutrients ${ }^{88-90}$. Unfortunately, the westernized lifestyle, consisting of a hypercaloric diet and limited exercise, downregulates AMPK and sirtuins. Caloric restriction, on the other hand, activates both pathways, stimulating efficient ATP production through glucose uptake and

Prog Mol Biol Transl Sci. Author manuscript; available in PMC 2019 February 01. 
glycolysis, fatty acid oxidation, and inducing recycling pathways to replenish energy ${ }^{91,}, 92$. AMPK is a structural sensor of the AMP/ATP ratio and low energy (high AMP) allows AMPK to be phosphorylated by its kinase LKB1. Pharmacologic activation of AMPK can be achieved by the anti-diabetic drug metformin, which will be used in the first life spanextension study in humans (TAME - targeting aging with metformin) ${ }^{4}, 93$. As a kinase, AMPK phosphorylates a number of target proteins to increase catabolic metabolism and inhibit mTOR. AMPK is a key mediator of longevity and induction of autophagy is an important downstream effect ${ }^{94}$. The recycling of cellular organelles increases lysosomal amino acid levels, which are detected by the mTOR complex. Amino acid-induced activation of mTOR then terminates the catabolic reaction in a homeostatic feedback loop.

Sirtuins are $\mathrm{NAD}^{+}$-dependent de-ac(et)ylases that exert several beneficial effects, such as detoxifying mitochondria predominantly via SIRT3 (see 2: Mitochondria and aging), increasing genomic stability through chromatin-associated SIRT6, and transcriptional activation of mitochondria by SIRT1 $24,95,96$. The additional function of sirtuins as ADPribosyltransferases is outside the scope of this review. With their dependence on $\mathrm{NAD}^{+}$, sirtuins effectively act as sensors for this coenzyme ${ }^{95}$. NAD ${ }^{+}$synthesis is under circadian control and can be quickly increased by fasting and AMPK activation. Importantly, NAD ${ }^{+}$ levels are depleted with age, partially through competition between sirtuins and other enzymes such as the $\mathrm{NAD}^{+}$-dependent DNA repair protein PARP ${ }^{4}$. Increased $\mathrm{NAD}^{+}$supply, on the other hand, has been shown to improve health and life span. Some of the immediate metabolic changes following $\mathrm{NAD}^{+}$repletion involve enhanced mitochondrial activity through deacetylation and activation of PGC1a by SIRT1, as well as improved mitochondrial proteostasis by SIRT3. Recently, SIRT7 has also been shown to improve mitochondrial health and aging in hematopoietic stem cells ${ }^{97}$.

\section{Coordination of nuclear activities and metabolism}

\section{The nuclear-metabolic axis}

Disruption of the nuclear architecture can have profound effects on aging, as shown by progeria-causing laminopathies. These diseases cause juvenile-onset aging, as in Hutchinson-Gilford Progeria Syndrome. Similar to DNA repair defects, affected patients age at an accelerated pace and die prematurely ${ }^{98,99}$. Laminopathies interfere with autophagy and impede mitochondrial activity through only partially understood mechanisms ${ }^{100}$. The mTOR inhibitor rapamycin has been shown to improve the cellular function of progeroid fibroblasts and treatment protocols are under development for these currently incurable diseases ${ }^{101}$.

Several pathways linked to cancer, specifically cell cycle control genes $\mathrm{p} 16^{\mathrm{INK} 4 \mathrm{a}}, \mathrm{p} 19^{\mathrm{ARF}}$ and the tumor suppressor $\mathrm{p} 53$, also play important roles in the aging process ${ }^{3,102-104}$. The expression levels of $\mathrm{p} 16^{\mathrm{INK} 4 \mathrm{a}}$ and $\mathrm{p} 19^{\mathrm{ARF}}$ correlate with age and using them as chronological markers has been proposed. Polymorphisms in these genes are associated with age-related diseases. Mild increases of $\mathrm{p} 16^{\mathrm{INK} 4 \mathrm{a}}, \mathrm{p} 19^{\mathrm{ARF}}$, and $\mathrm{p} 53$ have beneficial effects on aged cells, independent of their tumor-suppressive effects, but more significant upregulation accelerates aging. The mechanistic basis of this phenomenon remains unclear. 
Attrition of telomeres is a consequence of cell replication and aging. Shortened telomeres induce senescence and trigger a p53-dependent response that shuts down mitochondrial activity ${ }^{105}$. However, p53 can also increase mitochondrial output through association with the mitochondrial transcription factor TFAM in the matrix ${ }^{106}$. Therefore, key regulators of the cell cycle appear to have varied roles in metabolism and aging, depending on the context. Assembling a unifying model that explains all these activities poses a major challenge in this field.

\section{The metabolic-nuclear axis}

Studies on cancer metabolism and aging have converged on the link between the intermediary metabolism and epigenetics. DNA- and histone-modifying enzymes are key modifiers of the epigenetic landscape and require intermediary metabolites. There is growing interest in dissecting how metabolism affects gene expression and vice versa. One hallmark of aged cells is the loss of gene silencing ${ }^{107}$. Increased transcriptional noise exists due to a lack of heterochromatin, reactivation of transposons, dysregulated splicing, and other impairments ${ }^{108-111}$. In addition, epigenetic modifications can promote the inheritance of transgenerational longevity and metabolic traits in model organisms as well as in humans ${ }^{112-114}$. Among the epigenetic changes that occur during aging are loss of H3K9me3 modifications (a marker of gene repression), global loss of DNA methylation, and enhanced H4K16 acetylation (a marker of active genes) ${ }^{115-117}$. How these alterations are affected by the overall metabolic state and how they in turn regulate cell function is not entirely understood. Still, distinct metabolic pathways generate intermediates that are required for the enzymatic reactions that add or remove methyl or acetyl residues ${ }^{118-121}$.

The metabolites S-adenosyl methionine and acetyl-CoA are required for DNA and histone methylation, and for protein acetylation, respectively. Both are produced under nutrient-rich conditions. Their antagonists are alpha-ketoglutarate, which is required for protein and DNA demethylation and $\mathrm{NAD}^{+}$, a co-enzyme of sirtuin-dependent protein deacetylation. Both intermediates can increase under nutrient-low conditions: alpha-ketoglutarate levels are replenished by metabolic reactions that feed into the TCA cycle (anaplerosis) and $\mathrm{NAD}^{+}$ availability is regulated through AMPK-dependent synthesis ${ }^{122,123}$. While caloric restriction and elevated $\mathrm{NAD}^{+}$may attenuate aging by reducing protein acetylation, the effects on histone acetylation and gene activity are less obvious and await further clarification $^{124,125 .}$

$\mathrm{NAD}^{+}$also serves as a coenzyme of the DNA repair enzyme PARP and $\mathrm{NAD}^{+}$loss has been linked to genomic damage with age ${ }^{126}$. The TCA cycle intermediate alpha-ketoglutarate displays metabolic fluctuations. Hypoxia, for instance, favors the generation of its antagonist 2-hydroxyglutarate (L2HG), which inhibits demethylase activity ${ }^{127,} 128$. Formation of 2HG is also enhanced in certain cancers with mutations of the TCA enzyme IDH. These IDH mutations convert alpha-ketoglutarate into R2HG, which like L2HG blocks demethylation $^{129}$.

Epigenetic changes during aging affect the genome globally, but also locally. For instance, some genes defy global trends and are under direct regulation by metabolic pathways, such as FOXO target genes or stress response genes that are induced by ROS through the 
transcription factor NRF-2. Further, the cell cycle as well as senescence can be regulated by metabolism and mitochondrial activity through $\mathrm{p} 53^{130,131}$. For instance, under starvation AMPK phosphorylates p53 to induce p21, causing a cell cycle arrest. Mitochondrial stress in form of increased ROS levels also induces cell cycle arrest using mediators such as p53 and $\mathrm{p} 27^{130}$. Mitochondria can stimulate the expression of nuclear UPR ${ }^{\mathrm{mt}}$ genes through alterations in the epigenetic code ${ }^{132}$. It has been shown in worms that mitochondrial dysfunction increases histone demethylases to promote these UPR ${ }^{\mathrm{mt}}$ factors. Interestingly, to extend lifespan this effect needs to occur in the nervous system. The complex and sometimes contradictory effects of global and local epigenetic changes, as well as their connections with metabolism, chromatin, and aging defy summarization in a simple model ${ }^{133}$.

\section{Summary}

Genome-wide genetic studies in yeast and in worms have led to the discovery of the major pathways that govern longevity. Hormesis is a common principle across systems that applies to several of the described longevity pathways: limited metabolic impairment extends life span due to compensatory stress responses, whereas too much damage is harmful. Research findings based on blunt knock-out experiments may therefore require reevaluation in systems that allow for gene dosage adjustments. Still, the most exciting aspect of metabolism remains in the interconnectivity of pathways. How these different systems communicate with each other to synchronize their activity is the target of ongoing studies. Indeed, we are likely to make the most exciting discoveries about metabolic regulation of aging and find new avenues for therapeutic intervention at the intersection of these pathways.

\section{Acknowledgments}

This review was supported by the Cancer Prevention and Research Institute of Texas (RR140038), by the Ted Nash Long Life Foundation, and by NIH R01DK115454. We thank Catherine Gillespie for helpful comments and suggestions.

\section{References}

1. Cai W, et al. Oral glycotoxins are a modifiable cause of dementia and the metabolic syndrome in mice and humans. Proc Natl Acad Sci U S A. 2014; 111:4940-5. [PubMed: 24567379]

2. Hayflick L. Recent advances in the cell biology of aging. Mech Ageing Dev. 1980; 14:59-79. [PubMed: 7010011]

3. Lopez-Otin C, Blasco MA, Partridge L, Serrano M, Kroemer G. The hallmarks of aging. Cell. 2013; 153:1194-217. [PubMed: 23746838]

4. Lopez-Otin C, Galluzzi L, Freije JM, Madeo F, Kroemer G. Metabolic Control of Longevity. Cell. 2016; 166:802-21. [PubMed: 27518560]

5. Bjorkholm P, Harish A, Hagstrom E, Ernst AM, Andersson SG. Mitochondrial genomes are retained by selective constraints on protein targeting. Proc Natl Acad Sci U S A. 2015; 112:10154-61. [PubMed: 26195779]

6. Liu Z, Butow RA. Mitochondrial retrograde signaling. Annu Rev Genet. 2006; 40:159-85. [PubMed: 16771627]

7. Quiros PM, Mottis A, Auwerx J. Mitonuclear communication in homeostasis and stress. Nat Rev Mol Cell Biol. 2016; 17:213-26. [PubMed: 26956194]

8. Calvo SE, Mootha VK. The mitochondrial proteome and human disease. Annu Rev Genomics Hum Genet. 2010; 11:25-44. [PubMed: 20690818] 
9. Bratic A, Larsson NG. The role of mitochondria in aging. J Clin Invest. 2013; 123:951-7. [PubMed: 23454757]

10. Rose G, Santoro A, Salvioli S. Mitochondria and mitochondria-induced signalling molecules as longevity determinants. Mech Ageing Dev. 2016

11. Sack MN, Finkel T. Mitochondrial metabolism, sirtuins, and aging. Cold Spring Harb Perspect Biol. 2012; 4

12. Hohn A, Konig J, Grune T. Protein oxidation in aging and the removal of oxidized proteins. $\mathrm{J}$ Proteomics. 2013; 92:132-59. [PubMed: 23333925]

13. Sekhar RV, Liu CW, Rice S. Increasing glutathione concentrations with cysteine and glycine supplementation lowers inflammation in HIV patients. Aids. 2015; 29:1899-900. [PubMed: 26372395]

14. Nguyen D, Hsu JW, Jahoor F, Sekhar RV. Effect of increasing glutathione with cysteine and glycine supplementation on mitochondrial fuel oxidation, insulin sensitivity, and body composition in older HIV-infected patients. J Clin Endocrinol Metab. 2014; 99:169-77. [PubMed: 24081740]

15. Pathai S, Bajillan H, Landay AL, High KP. Is HIV a model of accelerated or accentuated aging? J Gerontol A Biol Sci Med Sci. 2014; 69:833-42. [PubMed: 24158766]

16. Jena NR. DNA damage by reactive species: Mechanisms, mutation and repair. J Biosci. 2012; 37:503-17. [PubMed: 22750987]

17. Suzuki YJ, Carini M, Butterfield DA. Protein carbonylation. Antioxid Redox Signal. 2010; 12:323-5. [PubMed: 19743917]

18. Trifunovic A, et al. Premature ageing in mice expressing defective mitochondrial DNA polymerase. Nature. 2004; 429:417-23. [PubMed: 15164064]

19. Kujoth GC, et al. Mitochondrial DNA mutations, oxidative stress, and apoptosis in mammalian aging. Science. 2005; 309:481-4. [PubMed: 16020738]

20. Perez VI, et al. The overexpression of major antioxidant enzymes does not extend the lifespan of mice. Aging Cell. 2009; 8:73-5. [PubMed: 19077044]

21. Chauhan A, Vera J, Wolkenhauer O. The systems biology of mitochondrial fission and fusion and implications for disease and aging. Biogerontology. 2014; 15:1-12. [PubMed: 24122214]

22. Muoio DM. Metabolic inflexibility: when mitochondrial indecision leads to metabolic gridlock. Cell. 2014; 159:1253-62. [PubMed: 25480291]

23. de Moura MB, dos Santos LS, Van Houten B. Mitochondrial dysfunction in neurodegenerative diseases and cancer. Environ Mol Mutagen. 2010; 51:391-405. [PubMed: 20544881]

24. Someya S, et al. Sirt3 mediates reduction of oxidative damage and prevention of age-related hearing loss under caloric restriction. Cell. 2010; 143:802-12. [PubMed: 21094524]

25. Scarpulla RC. Nuclear control of respiratory chain expression by nuclear respiratory factors and PGC-1-related coactivator. Ann N Y Acad Sci. 2008; 1147:321-34. [PubMed: 19076454]

26. Burnett C, et al. Absence of effects of Sir2 overexpression on lifespan in C. elegans and Drosophila. Nature. 2011; 477:482-5. [PubMed: 21938067]

27. Viswanathan M, Guarente L. Regulation of Caenorhabditis elegans lifespan by sir-2.1 transgenes. Nature. 2011; 477:E1-2. [PubMed: 21938026]

28. Saxena S, Caroni P. Selective neuronal vulnerability in neurodegenerative diseases: from stressor thresholds to degeneration. Neuron. 2011; 71:35-48. [PubMed: 21745636]

29. Dikic I. Proteasomal and Autophagy Degradation Systems. Annu Rev Biochem. 2017

30. Balchin D, Hayer-Hartl M, Hartl FU. In vivo aspects of protein folding and quality control. Science. 2016; 353:aac4354. [PubMed: 27365453]

31. Garcia-Prat L, Sousa-Victor P, Munoz-Canoves P. Proteostatic and Metabolic Control of Stemness. Cell Stem Cell. 2017; 20:593-608. [PubMed: 28475885]

32. Sadowska-Bartosz I, Bartosz G. Prevention of protein glycation by natural compounds. Molecules. 2015; 20:3309-34. [PubMed: 25690291]

33. Sala AJ, Bott LC, Morimoto RI. Shaping proteostasis at the cellular, tissue, and organismal level. J Cell Biol. 2017; 216:1231-1241. [PubMed: 28400444]

34. Vilchez D, Simic MS, Dillin A. Proteostasis and aging of stem cells. Trends Cell Biol. 2014; 24:161-70. [PubMed: 24094931]

Prog Mol Biol Transl Sci. Author manuscript; available in PMC 2019 February 01. 
35. Steffen KK, Dillin A. A Ribosomal Perspective on Proteostasis and Aging. Cell Metab. 2016; 23:1004-12. [PubMed: 27304502]

36. Holcik M. Could the eIF2alpha-Independent Translation Be the Achilles Heel of Cancer? Front Oncol. 2015; 5:264. [PubMed: 26636041]

37. Martinez G, Duran-Aniotz C, Cabral-Miranda F, Vivar JP, Hetz C. Endoplasmic reticulum proteostasis impairment in aging. Aging Cell. 2017

38. Shoulders MD, et al. Stress-independent activation of XBP1s and/or ATF6 reveals three functionally diverse ER proteostasis environments. Cell Rep. 2013; 3:1279-92. [PubMed: 23583182]

39. Taylor RC, Dillin A. XBP-1 is a cell-nonautonomous regulator of stress resistance and longevity. Cell. 2013; 153:1435-47. [PubMed: 23791175]

40. Glembotski CC. Endoplasmic reticulum stress in the heart. Circ Res. 2007; 101:975-84. [PubMed: 17991891]

41. Buttgereit F, Brand MD. A hierarchy of ATP-consuming processes in mammalian cells. Biochem J. 1995; 312(Pt 1):163-7. [PubMed: 7492307]

42. Russell JB, Cook GM. Energetics of bacterial growth: balance of anabolic and catabolic reactions. Microbiol Rev. 1995; 59:48-62. [PubMed: 7708012]

43. Schubert U, et al. Rapid degradation of a large fraction of newly synthesized proteins by proteasomes. Nature. 2000; 404:770-4. [PubMed: 10783891]

44. Hartl FU. Protein Misfolding Diseases. Annu Rev Biochem. 2017

45. McGuffee SR, Elcock AH. Diffusion, crowding \& protein stability in a dynamic molecular model of the bacterial cytoplasm. PLoS Comput Biol. 2010; 6:e1000694. [PubMed: 20221255]

46. Brehme M, et al. A chaperome subnetwork safeguards proteostasis in aging and neurodegenerative disease. Cell Rep. 2014; 9:1135-50. [PubMed: 25437566]

47. Calderwood SK, Murshid A, Prince T. The shock of aging: molecular chaperones and the heat shock response in longevity and aging--a mini-review. Gerontology. 2009; 55:550-8. [PubMed: 19546513]

48. Morrow G, Samson M, Michaud S, Tanguay RM. Overexpression of the small mitochondrial Hsp22 extends Drosophila life span and increases resistance to oxidative stress. Faseb J. 2004; 18:598-9. [PubMed: 14734639]

49. Walker GA, Lithgow GJ. Lifespan extension in C. elegans by a molecular chaperone dependent upon insulin-like signals. Aging Cell. 2003; 2:131-9. [PubMed: 12882326]

50. Voellmy R. On mechanisms that control heat shock transcription factor activity in metazoan cells. Cell Stress Chaperones. 2004; 9:122-33. [PubMed: 15497499]

51. Perez VI, et al. Protein stability and resistance to oxidative stress are determinants of longevity in the longest-living rodent, the naked mole-rat. Proc Natl Acad Sci U S A. 2009; 106:3059-64. [PubMed: 19223593]

52. Chondrogianni N, et al. Protein damage, repair and proteolysis. Mol Aspects Med. 2014; 35:1-71. [PubMed: 23107776]

53. Chondrogianni N, Petropoulos I, Franceschi C, Friguet B, Gonos ES. Fibroblast cultures from healthy centenarians have an active proteasome. Exp Gerontol. 2000; 35:721-8. [PubMed: 11053662]

54. Qian SB, McDonough H, Boellmann F, Cyr DM, Patterson C. CHIP-mediated stress recovery by sequential ubiquitination of substrates and Hsp70. Nature. 2006; 440:551-5. [PubMed: 16554822]

55. Segref A, et al. Pathogenesis of human mitochondrial diseases is modulated by reduced activity of the ubiquitin/proteasome system. Cell Metab. 2014; 19:642-52. [PubMed: 24703696]

56. Catic A, et al. Genome-wide map of nuclear protein degradation shows NCoR1 turnover as a key to mitochondrial gene regulation. Cell. 2013; 155:1380-95. [PubMed: 24315104]

57. Voos W, Rottgers K. Molecular chaperones as essential mediators of mitochondrial biogenesis. Biochim Biophys Acta. 2002; 1592:51-62. [PubMed: 12191768]

58. Lin YF, et al. Maintenance and propagation of a deleterious mitochondrial genome by the mitochondrial unfolded protein response. Nature. 2016; 533:416-9. [PubMed: 27135930] 
59. Bennett CF, Kaeberlein M. The mitochondrial unfolded protein response and increased longevity: cause, consequence, or correlation? Exp Gerontol. 2014; 56:142-6. [PubMed: 24518875]

60. Tian Y, Merkwirth C, Dillin A. Mitochondrial UPR: A Double-Edged Sword. Trends Cell Biol. 2016; 26:563-5. [PubMed: 27394966]

61. Wrobel L, et al. Mistargeted mitochondrial proteins activate a proteostatic response in the cytosol. Nature. 2015; 524:485-8. [PubMed: 26245374]

62. Taylor EB, Rutter J. Mitochondrial quality control by the ubiquitin-proteasome system. Biochem Soc Trans. 2011; 39:1509-13. [PubMed: 21936843]

63. Labbadia J, et al. Mitochondrial Stress Restores the Heat Shock Response and Prevents Proteostasis Collapse during Aging. Cell Rep. 2017; 21:1481-1494. [PubMed: 29117555]

64. Schon EA, Manfredi G. Neuronal degeneration and mitochondrial dysfunction. J Clin Invest. 2003; 111:303-12. [PubMed: 12569152]

65. Ruan L, et al. Cytosolic proteostasis through importing of misfolded proteins into mitochondria. Nature. 2017; 543:443-446. [PubMed: 28241148]

66. Bjedov I, et al. Mechanisms of life span extension by rapamycin in the fruit fly Drosophila melanogaster. Cell Metab. 2010; 11:35-46. [PubMed: 20074526]

67. Rubinsztein DC, Marino G, Kroemer G. Autophagy and aging. Cell. 2011; 146:682-95. [PubMed: 21884931]

68. Madeo F, Zimmermann A, Maiuri MC, Kroemer G. Essential role for autophagy in life span extension. J Clin Invest. 2015; 125:85-93. [PubMed: 25654554]

69. Farre JC, Subramani S. Mechanistic insights into selective autophagy pathways: lessons from yeast. Nat Rev Mol Cell Biol. 2016; 17:537-52. [PubMed: 27381245]

70. Pickrell AM, Youle RJ. The roles of PINK1, parkin, and mitochondrial fidelity in Parkinson's disease. Neuron. 2015; 85:257-73. [PubMed: 25611507]

71. Sarraf SA, et al. Landscape of the PARKIN-dependent ubiquitylome in response to mitochondrial depolarization. Nature. 2013; 496:372-6. [PubMed: 23503661]

72. Ho TT, et al. Autophagy maintains the metabolism and function of young and old stem cells. Nature. 2017; 543:205-210. [PubMed: 28241143]

73. Soubannier V, et al. A vesicular transport pathway shuttles cargo from mitochondria to lysosomes. Curr Biol. 2012; 22:135-41. [PubMed: 22226745]

74. Luo M, Zhao X, Song Y, Cheng H, Zhou R. Nuclear autophagy: An evolutionarily conserved mechanism of nuclear degradation in the cytoplasm. Autophagy. 2016; 12:1973-1983. [PubMed: 27541589]

75. Dou Z, et al. Autophagy mediates degradation of nuclear lamina. Nature. 2015; 527:105-9. [PubMed: 26524528]

76. Hohn A, Grune T. Lipofuscin: formation, effects and role of macroautophagy. Redox Biol. 2013; 1:140-4. [PubMed: 24024146]

77. Folick A, et al. Aging. Lysosomal signaling molecules regulate longevity in Caenorhabditis elegans. Science. 2015; 347:83-6. [PubMed: 25554789]

78. de Cabo R, Carmona-Gutierrez D, Bernier M, Hall MN, Madeo F. The search for antiaging interventions: from elixirs to fasting regimens. Cell. 2014; 157:1515-26. [PubMed: 24949965]

79. Heintz C, Mair W. You are what you host: microbiome modulation of the aging process. Cell. 2014; 156:408-11. [PubMed: 24485451]

80. Johnson SC, Rabinovitch PS, Kaeberlein M. mTOR is a key modulator of ageing and age-related disease. Nature. 2013; 493:338-45. [PubMed: 23325216]

81. Junnila RK, List EO, Berryman DE, Murrey JW, Kopchick JJ. The GH/IGF-1 axis in ageing and longevity. Nat Rev Endocrinol. 2013; 9:366-376. [PubMed: 23591370]

82. Salminen A, Kaarniranta K. AMP-activated protein kinase (AMPK) controls the aging process via an integrated signaling network. Ageing Res Rev. 2012; 11:230-41. [PubMed: 22186033]

83. Guarente L. Sirtuins in aging and disease. Cold Spring Harb Symp Quant Biol. 2007; 72:483-8. [PubMed: 18419308]

84. Martins R, Lithgow GJ, Link W. Long live FOXO: unraveling the role of FOXO proteins in aging and longevity. Aging Cell. 2016; 15:196-207. [PubMed: 26643314] 
85. Guo S, et al. Phosphorylation of serine 256 by protein kinase B disrupts transactivation by FKHR and mediates effects of insulin on insulin-like growth factor-binding protein-1 promoter activity through a conserved insulin response sequence. J Biol Chem. 1999; 274:17184-92. [PubMed: 10358076]

86. Houtkooper RH, Williams RW, Auwerx J. Metabolic networks of longevity. Cell. 2010; 142:9-14. [PubMed: 20603007]

87. Mihaylova MM, Shaw RJ. The AMPK signalling pathway coordinates cell growth, autophagy and metabolism. Nat Cell Biol. 2011; 13:1016-23. [PubMed: 21892142]

88. Fernandez-Marcos PJ, Auwerx J. Regulation of PGC-1alpha, a nodal regulator of mitochondrial biogenesis. Am J Clin Nutr. 2011; 93:884S-90. [PubMed: 21289221]

89. Price NL, et al. SIRT1 is required for AMPK activation and the beneficial effects of resveratrol on mitochondrial function. Cell Metab. 2012; 15:675-90. [PubMed: 22560220]

90. Kaur J, Debnath J. Autophagy at the crossroads of catabolism and anabolism. Nat Rev Mol Cell Biol. 2015; 16:461-72. [PubMed: 26177004]

91. Schaffer BE, et al. Identification of AMPK Phosphorylation Sites Reveals a Network of Proteins Involved in Cell Invasion and Facilitates Large-Scale Substrate Prediction. Cell Metab. 2015; 22:907-21. [PubMed: 26456332]

92. Singh R, Cuervo AM. Autophagy in the cellular energetic balance. Cell Metab. 2011; 13:495-504. [PubMed: 21531332]

93. Meng S, et al. Metformin activates AMP-activated protein kinase by promoting formation of the alphabetagamma heterotrimeric complex. J Biol Chem. 2015; 290:3793-802. [PubMed: 25538235]

94. Mair W, et al. Lifespan extension induced by AMPK and calcineurin is mediated by CRTC-1 and CREB. Nature. 2011; 470:404-8. [PubMed: 21331044]

95. Mostoslavsky R, et al. Genomic instability and aging-like phenotype in the absence of mammalian SIRT6. Cell. 2006; 124:315-29. [PubMed: 16439206]

96. Canto $\mathrm{C}$, et al. AMPK regulates energy expenditure by modulating NAD+ metabolism and SIRT1 activity. Nature. 2009; 458:1056-60. [PubMed: 19262508]

97. Mohrin M, et al. Stem cell aging. A mitochondrial UPR-mediated metabolic checkpoint regulates hematopoietic stem cell aging. Science. 2015; 347:1374-7. [PubMed: 25792330]

98. Carrero D, Soria-Valles C, Lopez-Otin C. Hallmarks of progeroid syndromes: lessons from mice and reprogrammed cells. Dis Model Mech. 2016; 9:719-35. [PubMed: 27482812]

99. Dobrzynska A, Gonzalo S, Shanahan C, Askjaer P. The nuclear lamina in health and disease. Nucleus. 2016; 7:233-48. [PubMed: 27158763]

100. Kubben N, et al. Repression of the Antioxidant NRF2 Pathway in Premature Aging. Cell. 2016; 165:1361-1374. [PubMed: 27259148]

101. Cao K, et al. Rapamycin reverses cellular phenotypes and enhances mutant protein clearance in Hutchinson-Gilford progeria syndrome cells. Sci Transl Med. 2011; 3:89ra58.

102. Krishnamurthy J, et al. Ink4a/Arf expression is a biomarker of aging. J Clin Invest. 2004; 114:1299-307. [PubMed: 15520862]

103. Jeck WR, Siebold AP, Sharpless NE. Review: a meta-analysis of GWAS and age-associated diseases. Aging Cell. 2012; 11:727-31. [PubMed: 22888763]

104. Dolivo D, Hernandez S, Dominko T. Cellular lifespan and senescence: a complex balance between multiple cellular pathways. Bioessays. 2016; 38(1):S33-44. [PubMed: 27417120]

105. Sahin E, et al. Telomere dysfunction induces metabolic and mitochondrial compromise. Nature. 2011; 470:359-65. [PubMed: 21307849]

106. Park JY, et al. p53 improves aerobic exercise capacity and augments skeletal muscle mitochondrial DNA content. Circ Res. 2009; 105:705-12. 11 p following 712. [PubMed: 19696408]

107. Pal S, Tyler JK. Epigenetics and aging. Sci Adv. 2016; 2:e1600584. [PubMed: 27482540]

108. Bahar R, et al. Increased cell-to-cell variation in gene expression in ageing mouse heart. Nature. 2006; 441:1011-4. [PubMed: 16791200] 
109. Huh I, Zeng J, Park T, Yi SV. DNA methylation and transcriptional noise. Epigenetics Chromatin. 2013; 6:9. [PubMed: 23618007]

110. Wood JG, Helfand SL. Chromatin structure and transposable elements in organismal aging. Front Genet. 2013; 4:274. [PubMed: 24363663]

111. Sedivy JM, et al. Death by transposition - the enemy within? Bioessays. 2013; 35:1035-43. [PubMed: 24129940]

112. Greer EL, et al. Transgenerational epigenetic inheritance of longevity in Caenorhabditis elegans. Nature. 2011; 479:365-71. [PubMed: 22012258]

113. Daxinger L, Whitelaw E. Transgenerational epigenetic inheritance: more questions than answers. Genome Res. 2010; 20:1623-8. [PubMed: 21041414]

114. Petersen KF, Dufour S, Befroy D, Garcia R, Shulman GI. Impaired mitochondrial activity in the insulin-resistant offspring of patients with type 2 diabetes. N Engl J Med. 2004; 350:664-71. [PubMed: 14960743]

115. Zhang W, et al. Aging stem cells. A Werner syndrome stem cell model unveils heterochromatin alterations as a driver of human aging. Science. 2015; 348:1160-3. [PubMed: 25931448]

116. Ashapkin VV, Kutueva LI, Vanyushin BF. Aging as an Epigenetic Phenomenon. Curr Genomics. 2017; 18:385-407. [PubMed: 29081695]

117. Taylor GC, Eskeland R, Hekimoglu-Balkan B, Pradeepa MM, Bickmore WA. H4K16 acetylation marks active genes and enhancers of embryonic stem cells, but does not alter chromatin compaction. Genome Res. 2013; 23:2053-65. [PubMed: 23990607]

118. Brunet A, Rando TA. Interaction between epigenetic and metabolism in aging stem cells. Curr Opin Cell Biol. 2017; 45:1-7. [PubMed: 28129586]

119. Chandel NS, Jasper H, Ho TT, Passegue E. Metabolic regulation of stem cell function in tissue homeostasis and organismal ageing. Nat Cell Biol. 2016; 18:823-32. [PubMed: 27428307]

120. Gut P, Verdin E. The nexus of chromatin regulation and intermediary metabolism. Nature. 2013; 502:489-98. [PubMed: 24153302]

121. Su X, Wellen KE, Rabinowitz JD. Metabolic control of methylation and acetylation. Curr Opin Chem Biol. 2016; 30:52-60. [PubMed: 26629854]

122. Wu N, et al. Alpha-Ketoglutarate: Physiological Functions and Applications. Biomol Ther (Seoul). 2016; 24:1-8. [PubMed: 26759695]

123. Canto C, Menzies KJ, Auwerx J. NAD(+) Metabolism and the Control of Energy Homeostasis: A Balancing Act between Mitochondria and the Nucleus. Cell Metab. 2015; 22:31-53. [PubMed: 26118927]

124. Peleg S, Feller C, Ladurner AG, Imhof A. The Metabolic Impact on Histone Acetylation and Transcription in Ageing. Trends Biochem Sci. 2016; 41:700-711. [PubMed: 27283514]

125. Peleg $\mathrm{S}$, et al. Life span extension by targeting a link between metabolism and histone acetylation in Drosophila. EMBO Rep. 2016; 17:455-69. [PubMed: 26781291]

126. Verdin $\mathrm{E} . \mathrm{NAD}(+)$ in aging, metabolism, and neurodegeneration. Science. 2015; 350:1208-13. [PubMed: 26785480]

127. Oldham WM, Clish CB, Yang Y, Loscalzo J. Hypoxia-Mediated Increases in L-2hydroxyglutarate Coordinate the Metabolic Response to Reductive Stress. Cell Metab. 2015; 22:291-303. [PubMed: 26212716]

128. Anso E, et al. The mitochondrial respiratory chain is essential for haematopoietic stem cell function. Nat Cell Biol. 2017; 19:614-625. [PubMed: 28504706]

129. Dang L, et al. Cancer-associated IDH1 mutations produce 2-hydroxyglutarate. Nature. 2009; 462:739-44. [PubMed: 19935646]

130. Lee IH, Finkel T. Metabolic regulation of the cell cycle. Curr Opin Cell Biol. 2013; 25:724-9. [PubMed: 23890700]

131. Wiley CD, et al. Mitochondrial Dysfunction Induces Senescence with a Distinct Secretory Phenotype. Cell Metab. 2016; 23:303-14. [PubMed: 26686024]

132. Merkwirth C, et al. Two Conserved Histone Demethylases Regulate Mitochondrial Stress-Induced Longevity. Cell. 2016; 165:1209-23. [PubMed: 27133168]

Prog Mol Biol Transl Sci. Author manuscript; available in PMC 2019 February 01. 
133. Han S, et al. Mono-unsaturated fatty acids link H3K4me3 modifiers to C. elegans lifespan. Nature. 2017; 544:185-190. [PubMed: 28379943] 


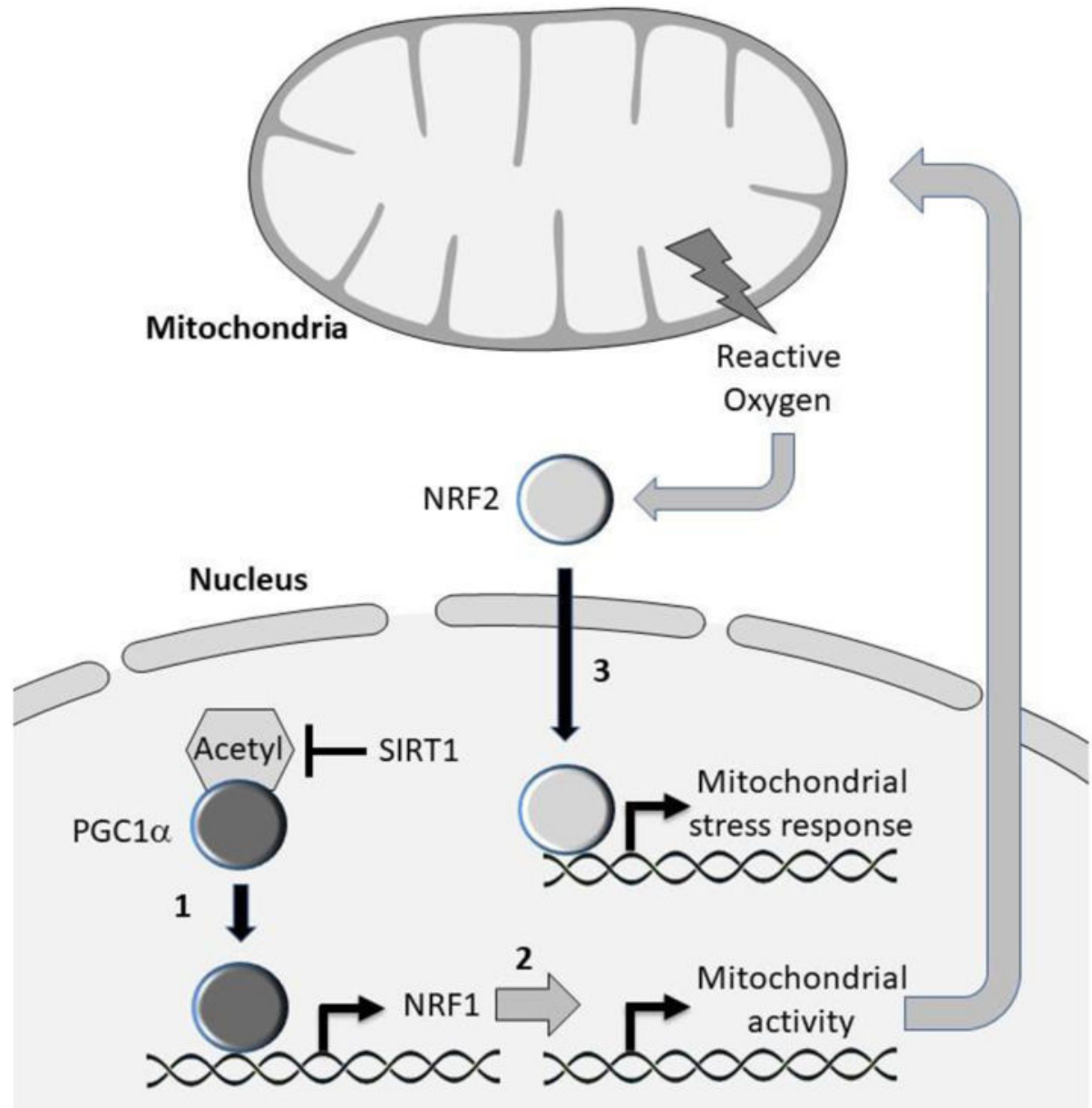

Figure 1. Master regulators of mitochondrial activity

SIRT1 activates expression of nuclear encoded mitochondrial genes through deacetylation of PGC1a. The sirtuins SIRT3, SIRT4, and SIRT5 reduce proteotoxic stress by removing protein acetylation in mitochondria (not shown). 


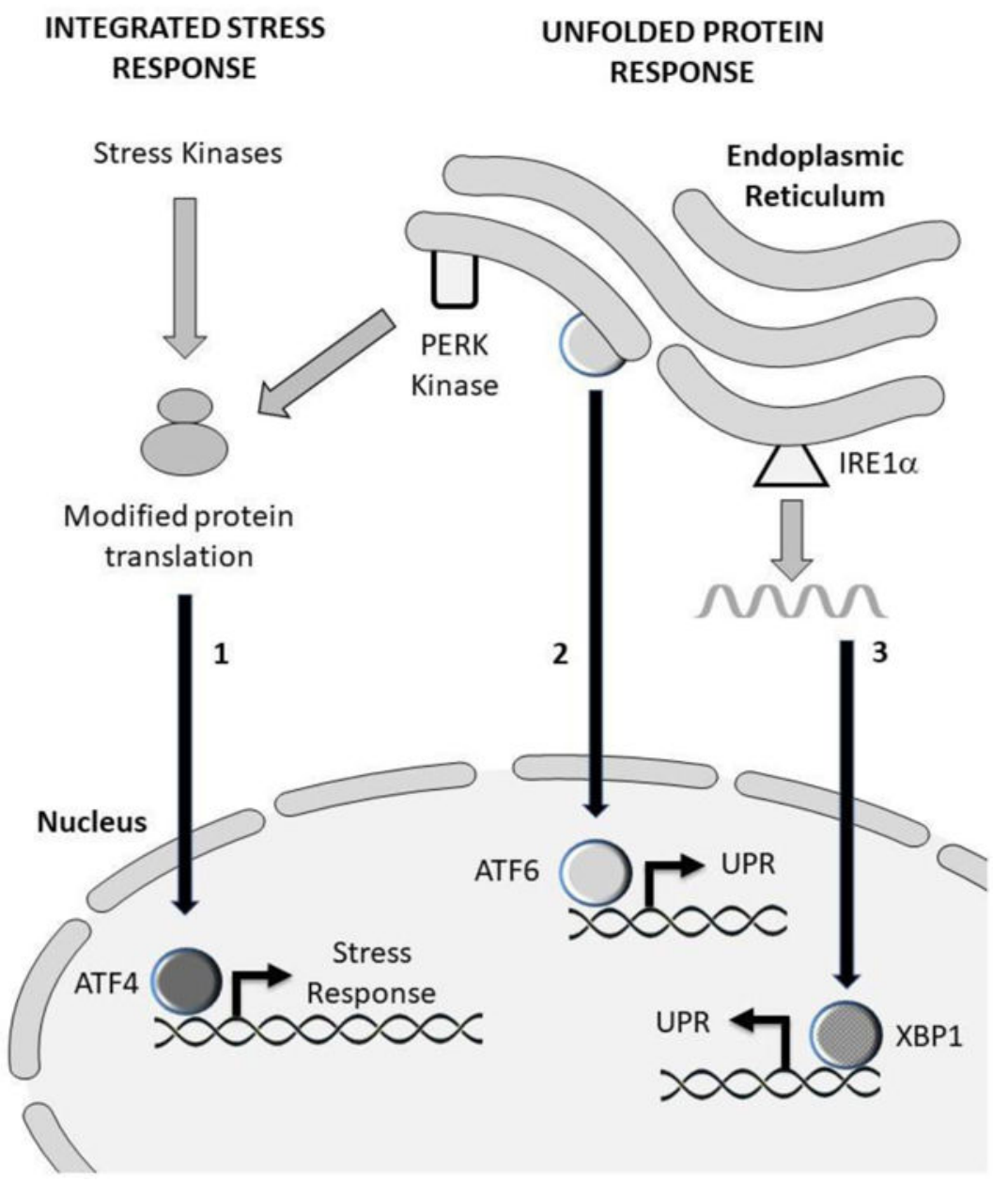

Figure 2. ISR and UPR effectors

Upstream stress kinases such as GCN2 and PKR, as well as the ER-bound kinase PERK, phosphorylate eIF2a This reduces overall protein translation, while favoring the expression of the transcription factor ATF4 and several chaperones. Additional elements of the UPR include the transcription factor ATF6, which translocates from the ER to the nucleus upon stress, and IRE1a, which allows for alternative splicing of the mRNA encoding the transcription factor XBP1, leading to its activation. 


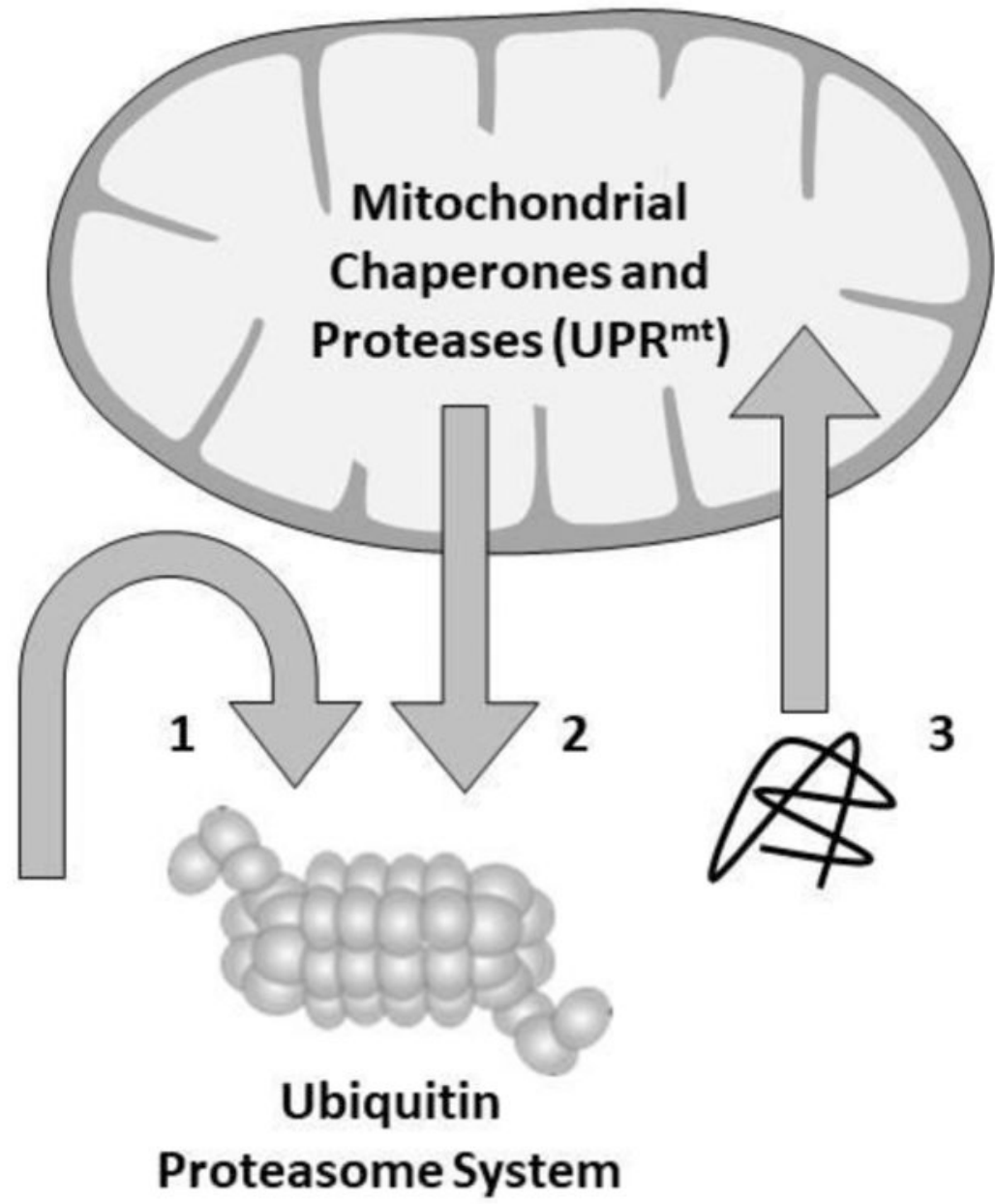

Figure 3. Cooperation between mitochondrial proteostasis and the UPS Mitochondrial proteins that fail to import into the organelle are removed by the UPS (1). Misfolded proteins can be extracted from mitochondria for proteasomal degradation, similar to the way misfolded proteins are extracted from the ER (2). Protein aggregates can be imported by mitochondria and destroyed by resident proteases (3). 


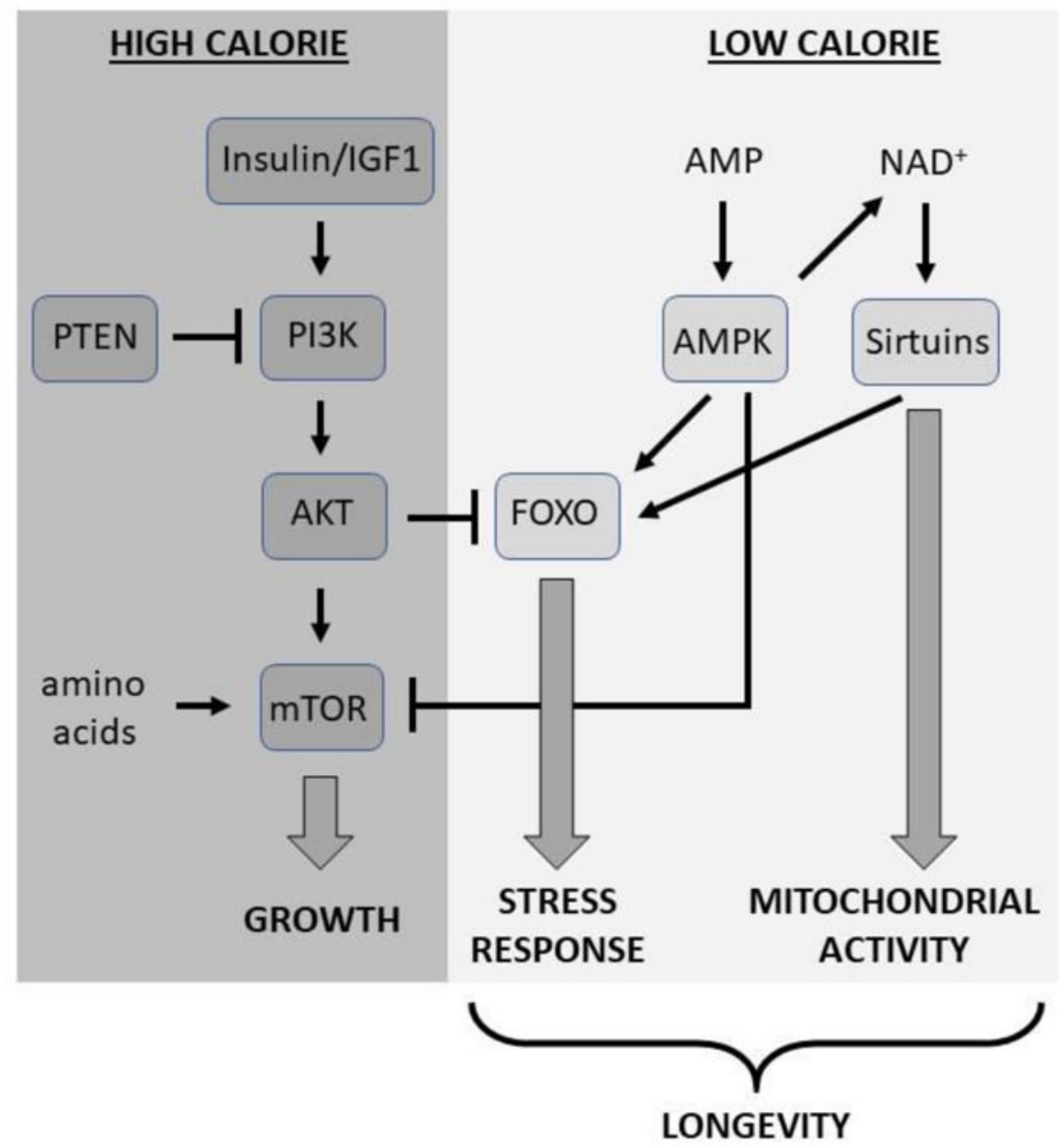

Figure 4. Nutrient-sensing pathways

Overview of the main pathways that sense sugar levels (insulin/IGF1) and amino acids (mTOR) or loss of bioavailable energy (AMPK) and NAD ${ }^{+}$depletion (sirtuins). High calorie conditions favor growth, while low calorie conditions improve the stress response, optimize energy metabolism, and have the potential to increase the life span. 